\title{
A micromechanical approach to describe internal erosion effects in soils
}

\author{
Luc Scholtes, Pierre-Yves Hicher \& Luc Sibille \\ GeM Laboratory, University of Nantes, ECN, CNRS, Nantes, France
}

\begin{abstract}
A multi-scale approach is proposed as an attempt to assess internal erosion induced effects on the mechanical properties of a granular medium. In this first analysis, the degradation of a granular assembly is simulated through the progressive removal of its smallest particles for both isotropic and anisotropic stress states. A discrete element model as well as a micromechanical constitutive relation is used to compare induced deformations and mechanical properties changes along the extraction process. The shear strength and flow properties appear to be strongly modified by this degradation: both models highlight the occurrence of failure during degradation process for stress states beyond the characteristic state; and, for unfailed samples, a change from a typical dilatant to a typical contractant behavior after degradation.
\end{abstract}

Keywords: erosion, micromechanics, porosity, volumetric strain, failure

\section{INTRODUCTION}

Internal erosion process can have a drastic influence on the behavior of granular soils by degrading their solid phase. For instance, the detachment and transport of some particles due to water internal flow induce strong modifications of the microstructure. For a complete description of internal erosion, one has to consider that solid elements can be extracted by water flow, whereas others may be relocated through the medium (Wan and Fell 2004). In zones where removal predominates, a challenging point is therefore to describe and understand the relation between the local degradation of the micro-structure and the resulting modification of the constitutive mechanical properties. In this paper, we present such an analysis through a multi-scale approach based upon numerical experiments performed with two fundamentally different models: a three-dimensional numerical model based on the Discrete Element Method, DEM (Cundall and Strack 1979), and an analytical model recently developed by Chang and Hicher (Chang and Hicher 2005) which can be viewed as a stress-strain relation built from a mechanical law between interparticle forces and displacements. Analyses are based on the simulated mechanical behavior of a granular assembly subjected to axisymmetric triaxial loadings. Starting from different initial stress states, particles the most likely erodible are progressively removed from the assembly. At the macroscopic scale, resulting strains and variations of mechanical properties are followed. An attempt to highlight the macroscopic mechanisms triggered by particle removals is suggested.

\section{MODELING THE EROSION PROCESS}

The aim of this study is not to provide an accurate estimation of internal erosion phenomena. Only a fully coupled hydro-mechanical model should be able to properly describe such a complex process. However, as the focus here is on the potential role of particle removals on the macroscopic response of a granular assembly, a simplified approach has been developed 
in order to mimic particle removal due to internal erosion. Both models are based on a similar homogenization process and can produce the macroscale behavior of a granular assembly from the occurrence of local forces and displacements at the particle level. In each case, the interparticle behavior follow an elastic law in the normal and tangential directions to the contact plane associated with Coulomb friction. DEM computations were performed through the YADE-Open DEM platform (Kozicki and Donze 2008). The numerical sample is a granular assembly composed of 10,000 spherical elements with an initial grading as presented in Fig. 1. During simulations, the stress-strain state of the specimen is controlled through the positions of six frictionless boundary walls such as in true triaxial tests.

DEM microscopic properties (see Table 1 for details) were chosen in order to produce a typical behaviour of a dense granular material, with strain softening associated to dilatancy. Fig. 2 shows the DEM model response when subjected to an axisymmetric triaxial compression under a $100 \mathrm{kPa}$ confining pressure. For consistency, the analytical model has been calibrated on the DEM one in order to simulate the same macroscopic behavior (Fig. 3). The following
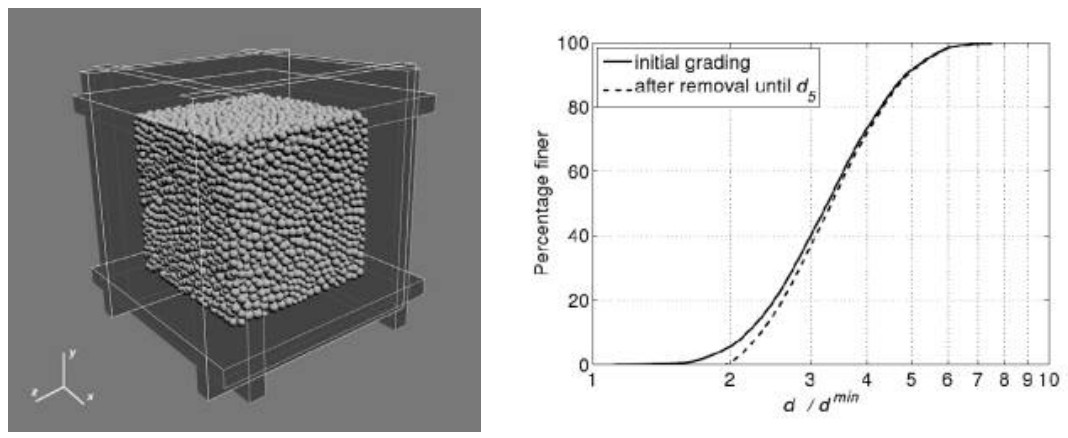

Figure 1. DEM numerical sample and particle size distribution before and after particle removal ( $\mathrm{d}$ indicates the sphere diameter).

Table 1. DEM parameters (d indicates the sphere diameter).

\begin{tabular}{lll}
\hline $\begin{array}{l}\text { Normal stiffness }(\mathrm{MPa}) \\
\mathrm{k}_{\mathrm{n}} / \mathrm{d}\end{array}$ & $\begin{array}{l}\text { Shear stiffness }(\mathrm{MPa}) \\
\mathrm{k}_{\mathrm{s}} / \mathrm{d}\end{array}$ & $\begin{array}{l}\text { Inter-particle friction angle }\left(^{\circ}\right) \\
\varphi\end{array}$ \\
\hline 250 & 125 & 35 \\
\hline
\end{tabular}
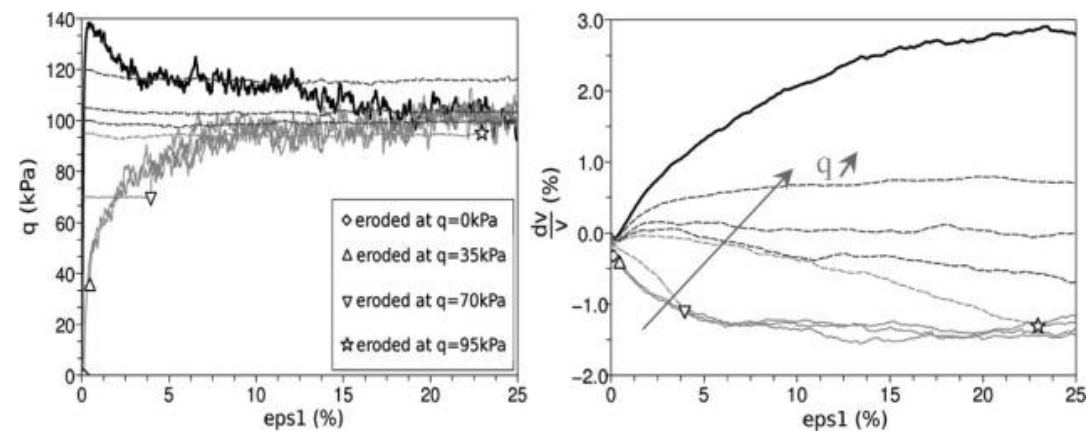

Figure 2. Summary of the simulated loadings applied to the DEM model under a $100 \mathrm{kPa}$ confining pressure. The thick line corresponds to the response of the intact specimen to a triaxial compression whereas thin lines correspond to the responses of the assembly when subjected to particle extraction for different stress ratios $q$ and to subsequent triaxial compressions when eroded samples have stabilized. Symbols indicate the transition between extraction and compression processes. 

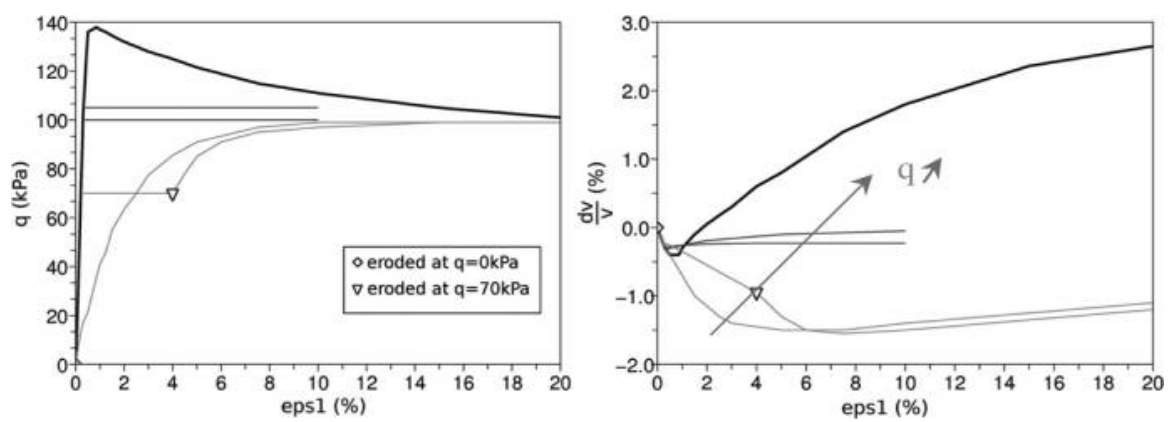

Figure 3. Summary of the simulated loadings applied to the microstructural model under a $100 \mathrm{kPa}$ confining pressure. The thick line corresponds to the response of the intact specimen to a triaxial compression whereas thin lines correspond to the responses of the assembly when subjected to particle extraction for different stress ratios $q$ and to subsequent triaxial compressions when eroded samples have stabilized. Symbols indicate the transition between extraction and compression processes.

sections describe how the modeling of the erosion process has been adapted and conducted on each model, depending on their respective constitutive parameters.

\section{$2.1 \quad D E M$}

Particle removal in the DEM model is based upon two assumptions. First, it can be properly assumed that seepage flow picks preferentially on the less constrained particles of the assembly. Second, particles should be small enough to be transported throughout the pore network. The less loaded particles belonging to the smallest particle fraction are thus the most likely erodible.

The magnitude of loading applied on each particle $p$ is estimated through its mean internal moment $m_{p}=\operatorname{tr}\left(\boldsymbol{M}_{p}\right)$, where $\boldsymbol{M}_{p}$ is the internal moment tensor of the particle $p$, as defined by Moreau (Moreau 1997), giving a good representation of stress at the particle scale. Particles are removed in a repetitive way from the assembly such as they have the smallest $m_{p}$ value among the $5 \%$ smallest particles in order to limit the extraction to the finest particles (Sibille and Scholtes 2009). During particle extraction, boundary wall positions are controlled in order to keep constant the prescribed stresses. Extraction is driven such as equilibrium is reached before any subsequent particle is removed. Between two particle extractions, the system evolves around quasi-static equilibrium with bursts of kinetic energy which intensity is linked to the role of the removed particle on the overall stability.

Fig. 2 presents the axial strains developing during particle removals initiated from different stress ratios $q=\sigma_{1}-\sigma_{2}$ along the triaxial loading path. Progressive strains develop within the specimen during particle extraction and tend to increase with $q$. It is remarkable that the degradation process initiates a contractancy of the sample for stress states prior to the characteristic state (corresponding approximately to $q \approx 100 \mathrm{kPa}$ ) and dilatancy for the upper stress states. Moreover, one can see that, when the erosion process is initiated beyond the characteristic state, it induces the failure of the specimen. Indeed, infinite strains develop and equilibrium is never reached for erosion processes initiated for $q \leq 100 \mathrm{kPa}$.

\subsection{Microstructural model}

As the basic idea of the model is to view the packing as represented by a set of micro systems which correspond to the contact planes, only the mean size value of the particles is considered as a model parameter. The model cannot take into account various sizes of individual particles, and it is, therefore, not possible to model the erosion process corresponding to the removal of the finest fraction as precisely as in the DEM approach. However, the number of inter-particle contacts can be reduced in a given assembly and the interlocking influence 
modified in touch with the sliding resistance at each contact. The sliding resistance has two origins: one corresponds to the friction between two particles defined by the internal friction angle; the other is linked to a geometrical effect of the neighboring particles, which will resist against the sliding displacement of two particles in contact and all the more so if the packing is dense. Therefore, the pulling off of a fraction of the particles will release the interlocking influence and facilitate the contacting particles to slide together. So, when part of the solid fraction is removed from the material, the void ratio increases and, as a consequence, the sliding resistance decreases, which can create additional inter-particle displacements. In this study, the extraction process is therefore simply considered through the removal of a given fraction of the solid particles. All the local displacements are then integrated to produce the macroscopic deformation of the soil specimen.

As presented in Fig. 3, despite the fundamentally different formulation of the microstructural model compared to the DEM one, their respective results are in very good agreement. Indeed, the strain increase during degradation with the level of the deviatoric stress, as well as the shifting from a contractant/stable to a dilatant/unstable behavior from the characteristic state is confirmed. The strain path followed during material degradation appears therefore totally linked to the mobilized friction level, and the characteristic state seems to give a good estimation of the limiting stress state beyond which a potential loss of stability of the medium can occur, for the applied extraction process.

\section{MATERIAL RESPONSES AFTER DEGRADATION}

In order to bring all the specimens to failure, triaxial compressions were continued for the samples which stabilized after the entire extraction process (see Fig. 2 and Fig. 3). Intuitively, the increase of the initial porosity produces a change in the specimen behavior from a dilatant behavior to a contractant behavior, confirming the pertinence of monitoring the porosity when considering mechanical properties of a potentially erodible material.

As shown previously, depending on the mobilized friction from which extractions are conducted, the sample tends to compress and stabilize or to dilate and fail, the limiting threshold being the characteristic state from which the material shifts from a stable to an unstable behavior. It is remarkable that this characteristic state is reached at the mobilized friction corresponding to the residual state obtained for large shear deformations of the initial intact specimen, the socalled critical state (Roscoe KH, Schofield AN, Wroth CP 1958). As defined in the Roscoe and Scoffield theory, at the onset of the critical state, shear distortions occur without any further changes in deviatoric stress or porosity. However, the value of the final porosity is different for the eroded specimens compared to the initial intact one as shown in Fig. 4.

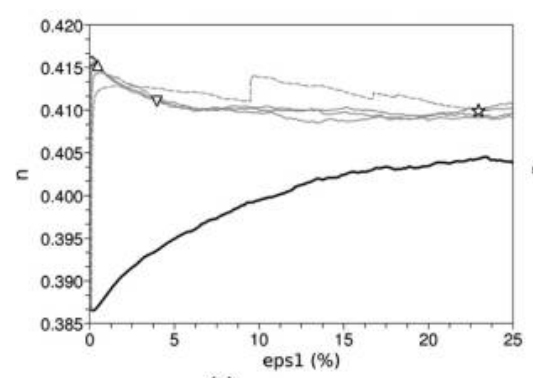

(a)

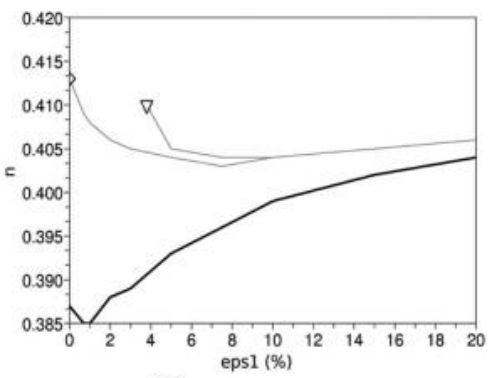

(b)

Figure 4. Evolution of the porosity during the simulated loadings applied to both the DEM model (a) and the microstructural model (b) under a $100 \mathrm{kPa}$ confining pressure. The thick lines corresponds to the response of the intact specimen to a triaxial compression whereas thin lines correspond to the responses of the assembly when subjected to particle extraction for different stress ratios $q$ and to subsequent triaxial compressions when eroded samples have stabilized. Symbols indicate the transition between extraction and compression processes. 
This is a remarkable issue which is probably due to the change of grading induced by particle removal. A granular assembly seems to seek out new critical state conditions as its grading changes as described by Muir-Wood and Maeda (Muir-Wood D, Maeda K 2008).

\section{CONCLUSION}

A multiscale approach to describe internal erosion consequences on the mechanical behavior of a granular assembly is proposed based on experiments realized upon two fundamentally different micromechanical models. As a first attempt to characterize internal erosion consequences on soil properties, results given by both approaches are in remarkable agreement. In particular, the results highlight a strong dependency of the strain path of the degraded material on its mobilized friction. This latter point suggests that failure or large strains can develop in hydraulic structures (such as dams or dykes) depending on the soil stress state in zones where internal erosion occurs, the critical state appearing to give the onset of failure. Besides, the results suggest that the change of porosity could be a valid parameter to describe internal erosion effects on the mechanical behavior of soils since this is the only parameter modified in the microstructural model. Finally, it appears that an assembly seeks out new critical state conditions when it suffers a change in its grading.

\section{ACKNOWLEDGEMENTS}

The scientific context and financial support provided by the French Region "Pays de la Loire" through the EMERMOD project is gratefully acknowledged.

\section{REFERENCES}

Chang CS, Hicher PY (2005). An elasto-plastic model for granular materials with microstructural consideration. Int. J. of Solids and Structures 42(12): 4258-4277.

Kozicki J, Donze FV (2008). A new open-source software developed for numerical simulations using discrete element modeling methods. Comput. Meth. Appl. to Mech. Eng. 197: 4429-4443. Moreau

JJ (1997). Numerical investigation of shear zones in granular materials. In D.E. Wolf, P. Grassenberger (eds), Friction, Arching, Contact Dynamics, World Scientific, Singapore: 233-247.

Muir-Wood D, Maeda K (2008). Changing grading of soils: effect on critical states. Acta Geotechnica 3(3): 3-14.

Roscoe KH, AN Schofield, CP Wroth (1958). On the Yielding of Soils. Geotechnique 8: 22-53. Sibille

L, Scholtes L (2009). Effects of internal erosion on mechanical behavior of soils: a DEM approach. Proceedings of Int. Conf. on Particle Based Methods, Particles 2009, Barcelona, Onate and D.R.J. Owen eds: $167-170$.

Wan CF, Fell R (2004). Experimental investigation of internal instability of soils in embankment dams and their foundations. UNICIV Rep. No. R 429, The University of NSW, Sydney. 